\title{
A FAST ALGORITHM AND TESTBED EVALUATION FOR SOUND-SOURCE LOCALIZATION USING SENSOR NETWORKS
}

\author{
Brian Johnston, Xiaoming Yin, Adrian Valenzuela, Patrick Frantz \\ Rice University \\ Dept. of Electrical and Computer Engineering \\ 6100 Main Street, Houston, TX 77005
}

\begin{abstract}
The theoretical idea of a network composed of small, low-cost sensor nodes able to localize the source of acoustic events has the potential to make a multitude of exciting applications possible. However, this potential can only be realized if computationally tractable algorithms are employed. In this paper, we propose a novel algorithm that offers not only low computational complexity, but also high accuracy, making it an excellent choice for implementation on low-power systems. Moreover, we have developed a flexible sensor network testbed and utilized it to determine the performance of our algorithm relative to existing methods. Finally, we present the results of our experimentation using real data collected with our sensor nodes, demonstrating the effectiveness of our localization scheme, and showing that our hardware platform is well-suited to the needs of sensor network applications.
\end{abstract}

\section{INTRODUCTION}

The ability to determine the source of an acoustic event and to distinguish such events from mere background noise could be extremely useful in many applications, especially if the task could be performed by small and inexpensive battery-powered sensor nodes. For instance, in a security application, the small devices could act as watchdogs for a large area and give an alert of any disturbance, as well as its calculated location.

In order for a localization algorithm to be implemented on real sensor network hardware, the procedure must be computationally tractable for low-power sensor nodes. Therefore, we propose a new localization algorithm which exploits the relationship between the maximum-likelihood estimate of the sound-source location and the underlying geometry of the sensor arrangement. The algorithm not only has a low computational complexity (depending only on the number of sensors deployed), but also boasts accuracy approaching that of an exhaustive search, as seen in the results of our experimentation (with real data), illustrated in Figure 5 and summarized in Table I.

Further, we created a flexible sensor network testbed, described in section IV on which to implement the algorithm, allowing us to test its performance on real data. We used this platform to perform a comparative analysis of our localization algorithm with existing methods. In addition to demonstrating the effectiveness of our algorithm, the experimentation showed our platform to be well-suited to the needs of sensor network applications.
Many algorithms exist for converting the raw data from the sensors into the event's coordinates (such as time delay of arrival [1] [2] and direction of arrival [3] [4]), but one that seems particularly well-suited to low-power sensor networks is an energy-based calculation [5] [6]. In our work, we have utilized the maximum likelihood energy-based model described in [5], summarized here for the convenience of the reader. Although the model allows for multiple sound-sources, we will focus on the single-source case.

In developing and employing any such model, numerous simplifying assumptions are made. In our testing, we designed our experiments to largely conform to these assumptions, and therefore it is not terribly surprising that the algorithm performs well. However, having demonstrated through our experiments that the algorithm is both computationally viable and largely accurate when our assumptions hold, we are now beginning to perform experiments to learn what happens as these assumptions begin to break down. Using our hardware testbed, we hope to find ways to refine the theoretical underpinnings of the algorithm in order to better accommodate circumstances that deviate from the model's expectations.

First, section II describes the data model underlying the localization algorithm. Next, in section III, we present our algorithm for maximizing the likelihood function with reduced complexity. Section IV details our sensor network hardware platform. We then describe our experiments (section V), and our results (section VI). Finally, section VII concludes the paper.

\section{DATA MODEL}

The underlying assumption of the algorithm is that acoustic energy is inversely proportional to the square of the distance from the source [7]. This implies that we are treating the sound-source as an omni-directional point source. We assume that the signal received at the $i$ th sensor $(i=1,2, \ldots N)$ at time $t$ will be:

$$
x_{i}(t)=\frac{g_{i} \cdot s(t)}{d_{i}^{2}(t)}+w_{i}(t)
$$

where $g_{i}$ is the microphone gain at the $i$ th sensor, $s(t)$ is the signal one unit-distance away from the source, $d_{i}^{2}(t)$ is the 
Euclidean distance between the source and the $i$ th sensor in the same units, and $w_{i}(t)$ is the observation noise at the $i$ th sensor, modeled as a zero-mean Gaussian random variable.

Since the energy for most sources will be slowly varying relative to the sampling rate, we can get a better estimate by averaging the energy over $\mathrm{T}$ samples. Thus, the input to the algorithm will be:

$$
y_{i}(t)=\frac{1}{T} \sum_{t=0}^{T-1} \frac{g_{i}^{2}(t) \cdot s^{2}(t)}{d_{i}^{2}(t)}+\frac{1}{T} \sum_{t=0}^{T-1} w_{i}^{2}(t)
$$

Because the use of these time averages is permissible, the dimensionality of the data that must be transmitted from the nodes is significantly reduced from the actual acoustic time series. This reduces the amount of data that must be communicated from the nodes and thus the overall power consumption.

\section{LOCALIZATION AlgorithM}

Let the vector $\mathrm{Z}$ contain the average energy readings from each sensor for one time window.

$$
Z=\left[\begin{array}{llll}
y_{1} & y_{2} & \ldots & y_{N}
\end{array}\right]^{T}
$$

Let the vector $\mathrm{H}$ contain the gain of each sensor divided by the distance from the sensor to source squared.

$$
H=\left[\begin{array}{llll}
\frac{g_{1}}{d_{1}^{2}} & \frac{g_{2}}{d_{2}^{2}} & \cdots & \frac{g_{N}}{d_{N}^{2}}
\end{array}\right]^{T}
$$

As derived in [5], the maximum likelihood estimate of the source coordinates will minimize the negative-log likelihood function:

$$
L^{\prime}(\theta)=Z^{T} Z-Z^{T} P_{H} Z
$$

where $P_{H}$ is a projection matrix given by:

$$
P_{H}=\frac{1}{H^{T} H} H H^{T}
$$

and $\theta$ is a vector containing the unknown source coordinates. Since only the second term involves the coordinates, thus minimizing the expression above is equivalent to maximizing the second term:

$$
L(\theta)=Z^{T} P_{H} Z
$$

Note that this cannot be solved directly, as $P_{H}$ is a non-linear function of the unknown source coordinates. Therefore, we use numerical methods to maximize the likelihood function. The first two methods presented here are taken from [5], but the third is a novel approach.

\section{A. Exhaustive Search}

Although we will discuss source localization in two dimensions, the discussion can easily be generalized to arbitrarily many dimensions. In this scenario, the likelihood function can be thought of as a three-dimensional surface having some height for each $(x, y)$ pair. The exhaustive search method samples the surface using an arbitrarily fine grid and should be able to find a point very close to the absolute maximum if the resolution is high enough. However, the computational complexity of this method is quite high. If we choose to sample $\mathrm{M}$ evenly-spaced points in each dimension, then the complexity is $O\left(M^{2}\right)$. For any reasonable resolution and search area this complexity will quickly become intractable for low-power hardware operating in real-time. Clearly, an optimized approach which does not evaluate all points is needed.

\section{B. Grid-based Multi-resolution Search}

The multi-resolution search proposed in [5] would only examine $m$ points in each dimension per iteration, choose the largest of those, and then narrow the search to the region around the point which gave rise to the higher value of the likelihood function. Thus, after $\log _{m} M$ iterations, the resolution of the search will be the same as the exhaustive search. Furthermore, the complexity is reduced to $O(\log M)$.

Although the complexity reduction is considerable, it comes at a price. Because the search grid is so coarse at earlier stages, it is quite conceivable that the true maximum "hill" in the surface could be missed and a smaller one (or local maximum) could be identified as the maximum likelihood location instead. In our experiments, this had the result of increasing the mean and variance of the estimation error as compared to the exhaustive search.

\section{Sensor Geometry-based Multi-resolution Search}

While a multi-resolution search makes a lot of sense, it seems that the method of choosing the points to evaluate should take into account the specific nature of the likelihood function we are evaluating and where it comes from. For instance, the surface of the likelihood function is generated using the received energy values and primarily has to do with the distances between the source and each sensor. It becomes clear after looking at a number of representative likelihood functions that the shape of the surface has a lot to do with the underlying geometry of the sensor arrangement. Additionally, the maximum of the likelihood function will be closer to the sensor with the highest energy reading than to any other sensor, and thus it seems unnecessary to stray too far from this area. These considerations motivated the following approach.

1) Algorithm: Let the $N \times 2$ matrix $S$ contain the $(x, y)$ coordinates of the $\mathrm{N}$ sensors. 


$$
S=\left[\begin{array}{cc}
x_{1} & y_{1} \\
x_{2} & y_{2} \\
\vdots & \vdots \\
x_{N} & y_{N}
\end{array}\right]
$$

Additionally, let $S_{\max }$ be a row vector (taken from $S$ ) containing the $(x, y)$ coordinates of the sensor with the highest energy reading, and let $P_{i, j}$ be a row vector containing the $(x, y)$ coordinates of the $j$ th point to evaluate on the $i$ th iteration. For the first iteration, $P_{1, j}$ is given by:

$$
P_{1, j}=\frac{S_{j}+S_{\max }}{2} \forall S_{j} \neq S_{\max }
$$

Thus, the mid-point between the location of the sensor with the maximum energy reading and the location of every other sensor become the points selected (for $S$ sensors, you will select $S-1$ points). Figure 1(a) illustrates this process (the circles represent $S_{j}$, the 'x' marks the sound-source location, and the asterisks indicate $P_{1, j}$, the points selected in the first iteration according to equation 9). Then, evaluate the likelihood function at those points and select the maximum. This point will be called $P_{1, \max }$. For all subsequent iterations, the points to be evaluated will be given by:

$$
P_{i, j}=\frac{P_{i-1, j}+P_{i-1, \max }}{2} \forall P_{i-1, j} \neq P_{i-1, \max }
$$

Figure 1(b) illustrates this for iteration 2 (the circles represent $P_{1, j}$, the ' $\mathrm{x}$ ' marks the sound-source location, and the asterisks indicate $P_{2, j}$, the points selected in the second iteration according to equation 10 . Note that each iteration there will be one less point evaluated than in the previous iteration. This process can be repeated as many times as desired for increased precision, up to $N-1$ iterations.

2) Variations: There are two slight modifications that can optionally be made. First, additional points can be evaluated on the first iteration for increased precision. For each point $P_{i, j}$ already selected, choose $c$ additional points lying on the line between the sensor with the highest energy reading $\left(S_{\max }\right)$ and the points already selected $\left(P_{i, j}\right)$. This would probably be a good idea if the sensors are quite far apart. If it is only done on the first iteration then the number of points selected will be $(c+1) \cdot(S-1)$ initially and one less for each iteration thereafter. Thus, the complexity is only increased by the constant $c+1$.

Second, the algorithm as described above will only search points bounded by the sensors, thus if the source is outside of the sensor field the estimate will not be correct. In many cases, this may be acceptable as one would primarily expect sources to be within the field, or else coverage should probably be expanded. (Through analysis of the Cramer-Rao Lower Bound, it is shown in [5] that the variance of the estimate is lowest when the source is close to many sensors, which occurs when the source is in the midst of the sensors.)

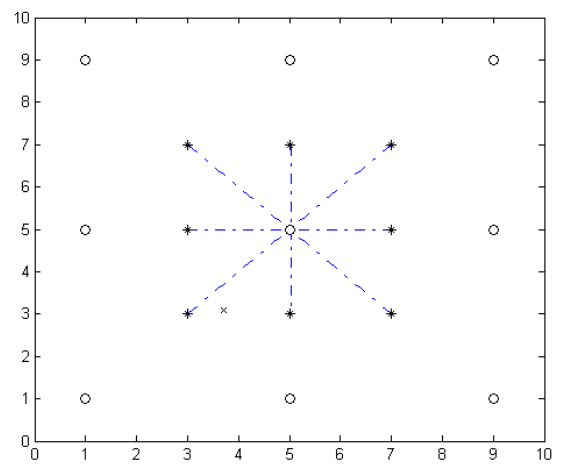

(a) Iteration 1

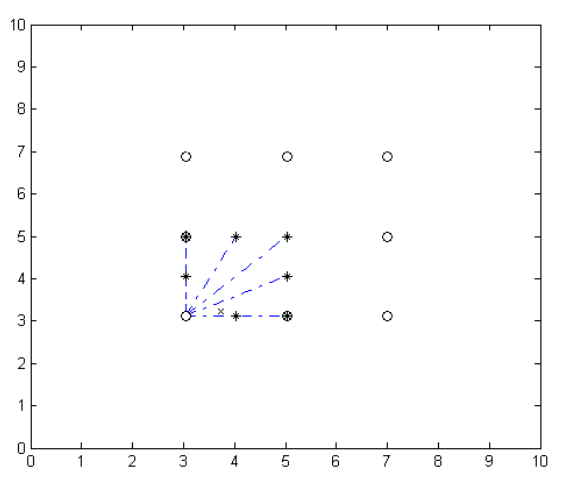

(b) Iteration 2

Fig. 1. Illustration of Sensor Geometry-based MR Search

However, a small modification can be made to the search to allow points not bounded by the sensors to be searched. In each iteration, determine the points to be searched using the method originally described in section III-C.1. Then, for each point $P_{i, j}$ already selected choose $c$ additional points having the same radius from the maximum point $\left(P_{i-1, \max }\right)$, but an angle between 90 and 270 degrees (with respect to the center of the circle $P_{i-1, \max }$ ) from the point $P_{i, j}$ originally selected. (If $c=1$, or instance, it would make sense to select the point on the opposite side of the circle, at an angle of 180 degrees with respect to the center of the circle.) This will allow searching in directions away from the rest of the sensors, thus extending outside the area bounded by the sensors. Now $(c+1) \cdot(S-1)$ points will be chosen on the first iteration, $(c+1) \cdot((c+1) \cdot(S-1)-1)$ points will be chosen in the second iteration, and so on.

3) Complexity: Any of the three formulations of this method presented offer complexity $O(S)$, where $S$ is the number of sensors. This complexity is low compared to the exhaustive search and similar to the multi-resolution search described in the last section, since in general $S$ will be much smaller than $M$. Of the three formulations, the first will have the lowest complexity, while the last will have the highest complexity.

The other advantage this method has over the grid-based 


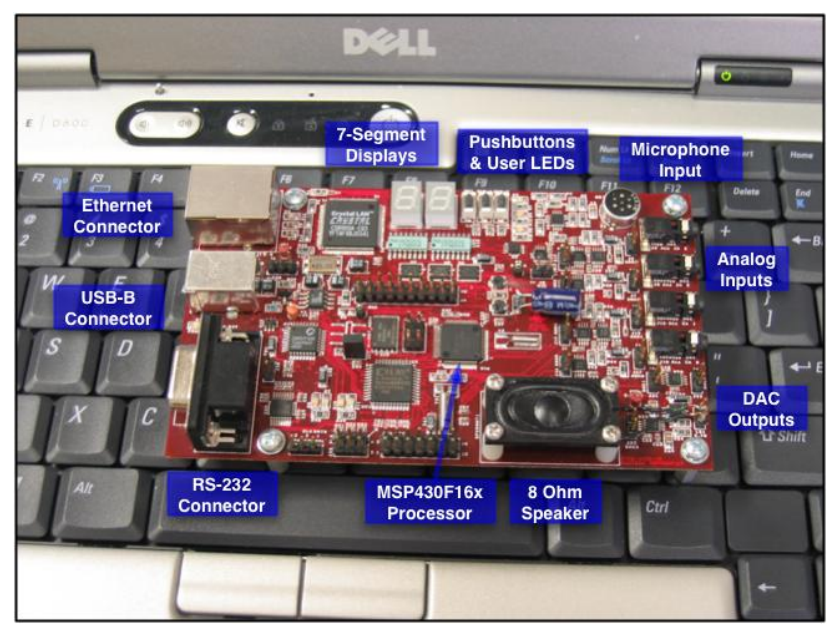

Fig. 2. MSP430F169DLX Hardware Development Platform

multi-resolution search described previously is that in our tests it has proved to be far less likely to be trapped in a local maximum, although admittedly it is still possible since it is not an exhaustive search. In our experiments, the accuracy was comparable to the exhaustive search, while the complexity was on par with the grid-based multi-resolution search. Therefore, we suggest that this optimized search is an excellent choice for implementation in low-power sensor nodes.

\section{HARDWARE PLATFORM}

In order to compare the performance of our algorithm to that of existing methods, we needed a flexible hardware platform that would allow us to quickly and easily implement and test those algorithms. Based on our design of and work with the sensor network platform GNOMES [8], we designed the custom MSP430F169DLX development board shown in Figure 2 , to give us extra flexibility and features. Originally designed as a development board for an introductory micro-controller course at Rice, ELEC 226 [9], the MSP430F169DLX board expands on the integrated peripheral set the GNOMES platform provides. Given that the MSP430F169DLX platform was mainly to be used in a lab environment, wired communication was given precedence over wireless communication. Also, power was not used as a design consideration when adding functionality and flexibility.

From the MSP430F169DLX board block diagram, as seen in Figure 3, one can note the upgrade of 16-bit microcontroller from the MSP430F149, used on GNOMES, to either the MSP430F169, with 60KB of flash code memory and $2 \mathrm{~KB}$ of SRAM data, or the MSP430F1611, with $48 \mathrm{~KB}$ of flash code memory and $10 \mathrm{~KB}$ of SRAM data. This upgrade allowed for greater data movement via the internal DMA as well as integrated digital to analog conversion. The focus on wired communication can also be seen by the integration of both 10 Base-T Ethernet and RS-232 Serial PC Interface. A wireless communication system has not been integrated on the MSP430F169DLX hardware platform but can be added later as a plug-in module. Finally, the block diagram shows the wide

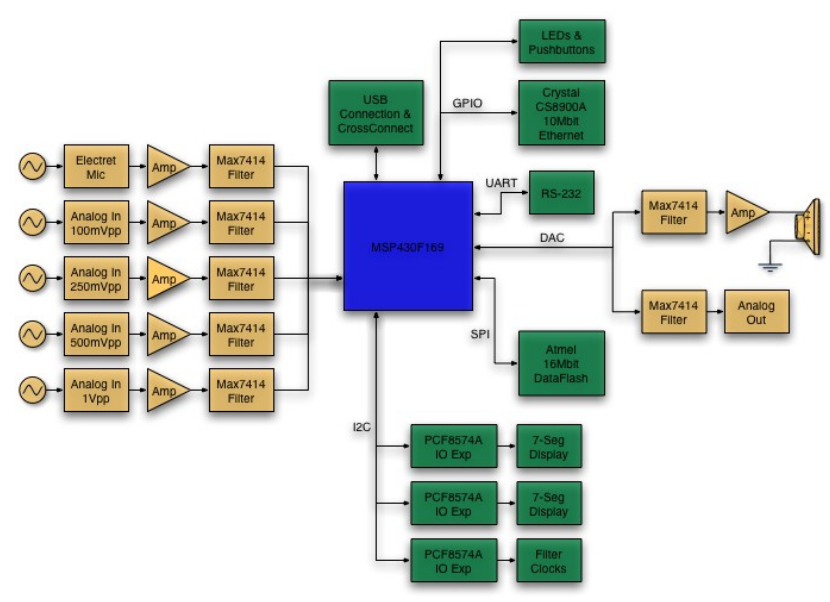

Fig. 3. Block Diagram of Hardware Development Platform

array of both analog and digital integrated peripherals which include: an embedded USB Cross-Connect JTAG emulator; 2MBytes of SPI DataFlash; up to 16 user-accessible digital I/Os; dual 7-segment displays; 2 de-bounced user pushbuttons with interrupt capability; 3 user LEDs; electret microphone input; 4 optionally amplified analog inputs; programmable preADC anti-alias filtering using the MAX7414, a 5th order lowpass Butterworth filter with a tunable $f_{c}$ from $1 \mathrm{~Hz}$ to $15 \mathrm{kHz}$; 2 DAC outputs, one with optional $0.7 \mathrm{~W}$ amplification to an 8 Ohm speaker; programmable post-DAC filtering using the MAX7414; and system current measurement.

All the parts for the MSP430F169DLX hardware platform are standard off-the-shelf components, making the platform both affordable and easy to build. The diverse peripheral set and communication options allow for a wide variety of sensor network applications to be developed for the platform.

\section{EXPERIMENTS}

The sound localization algorithm was implemented on the hardware platform described in section IV. Fifty tests were conducted on an indoor, $25 \times 17$ foot grid. Over the course of each test, several hundred estimates of the sound-source location were made. Tested node arrangements include the following: nodes at right angles using various inter-node spacing, random scattering, circular shapes, and a linear arrangement. For each sensor arrangement, experiments were run using a stationary sound source (moved for each trial), a moving source, and a stationary source with significant amounts of ambient noise. The data used to compute the results in section VI is drawn from all tested sensor arrangements.

Nine sensor nodes were used during testing. Once activated by the master node, each sensor node would sample sound data received through the on-board microphone and compute the energy. The average energy over 64 samples was transmitted every $6 \mathrm{~ms}$ via Ethernet to a computer serving as the master node, where the location was computed using all three methods described in section III in order to compare their performance.

A computer was chosen to serve as the master node in these experiments because the Exhaustive Search could not be 


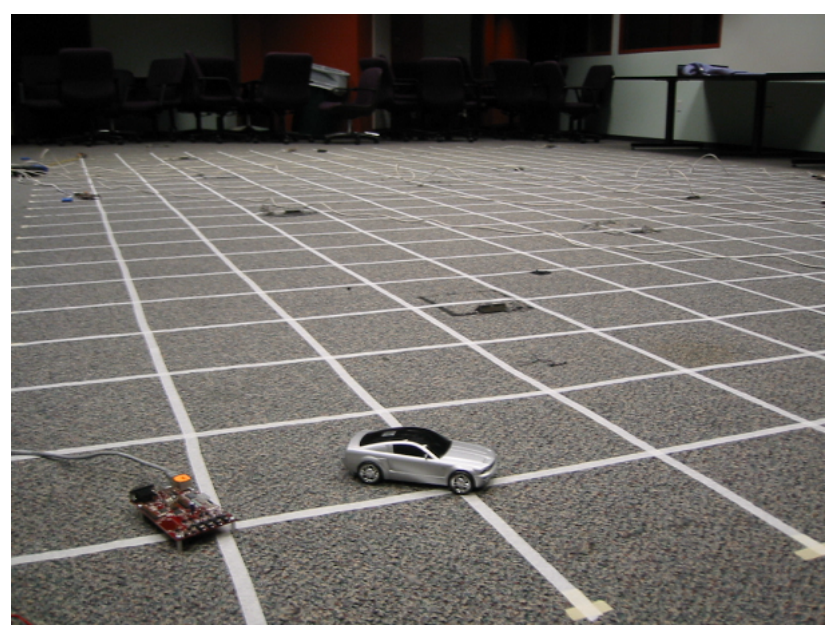

Fig. 4. Experimental Setup

performed in real-time on the nodes, due to its prohibitively high complexity. Therefore, the computer was used in order to ensure a balanced comparative analysis. The complexity of the MR algorithms is low enough to permit real-time processing on a node, and the accuracy of the algorithm is not platformdependent. Thus, it seems reasonable to expect similar performance when the data analysis is done in hardware.

\section{RESULTS}

Figure 5(a) shows a plot verifying the validity of the energy attenuation model, showing the received energy reading vs. distance in feet from the sound-source. The y-axis is logarithmic to highlight deviations from the model. The dotted line indicates the model prediction, while the solid line indicates the empirical data. While the model is not perfect (due in part to the omni-directional point-source assumption), the shape of the curve is generally correct.

Figure 5(b),(c), and (d) show histograms of the estimation error (in feet) for the Exhaustive Search, Grid-based MR Search, and Sensor Geometry-based MR Search, respectively, allowing for a comparison of the accuracy of the three methods presented in section III. Of the three, 5(c) has the heaviest tail, indicating that the Grid-based MR Search is more likely to yield estimates far from the correct location.

The results for each method are summarized in Table I. The Exhaustive Search with its high computational complexity is the standard against which the two lower-complexity methods must be judged. Clearly, of the two, the Sensor Geometrybased MR Search is the better method, yielding an average error and variance rivaling the Exhaustive Search.

\section{CONCLUSION}

We have proposed a novel algorithm for sound-source localization in sensor networks and demonstrated it to be comparable in accuracy to an exhaustive search, but with a greatly reduced computational complexity. Further, we have developed a sensor network testbed on which we evaluated our

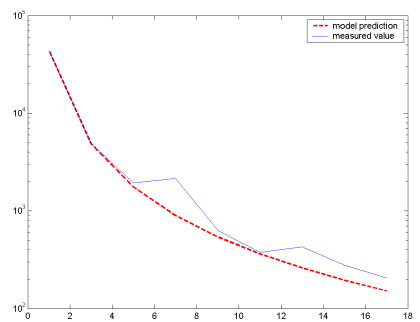

(a) Energy Attenuation Model

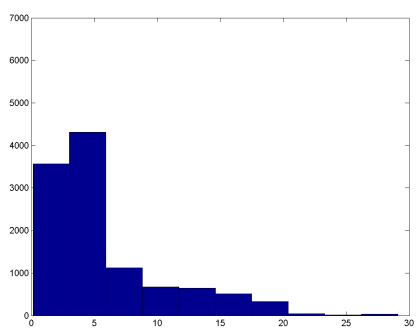

(c) Grid MR Search Error

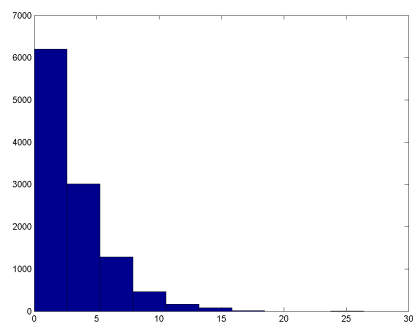

(b) Exhaustive Search Error

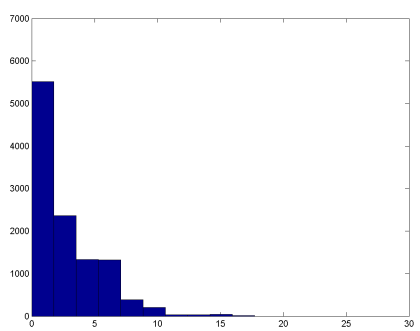

(d) Sensor Geo. MR Search Error
Fig. 5. Experimental Results

\begin{tabular}{l|r|r|r} 
Method & Avg. Error & Var. of Error & Complexity \\
\hline Exhaustive & $3.02 \mathrm{ft}$. & $6.34 \mathrm{ft}$. & $O\left(N^{2}\right)$ \\
Grid MR & $5.76 \mathrm{ft}$. & $23.06 \mathrm{ft}$. & $O(\log N)$ \\
Sensor MR & $3.09 \mathrm{ft}$. & $6.58 \mathrm{ft}$. & $O(S)$ \\
& & &
\end{tabular}

COMPARISON OF SEARCH AlgorithmS

algorithm and, in so doing, demonstrated the suitability of our hardware platform for real-world sensor network applications.

\section{ACKNOWLEDGMENT}

The authors wish to thank Erik Welsh and Dr. Ashutosh Sabharwal for their valuable advice and contributions regarding this manuscript.

\section{REFERENCES}

[1] R. Schmidt, "Least squares range difference location," IEEE Trans. Aerospace and Electronic Sys., vol. 32, no. 1, pp. 234-242, Jan. 1996.

[2] J. Smith and J. Abel, "Closed-form least-squares source location estimation from range-difference measurements," IEEE Trans. ASSP, vol. 35, no. 12, pp. 1661-1669, Dec. 1987.

[3] Y. Oshman and P. Davidson, "Optimization of observer trajectories for bearings-only target localization," IEEE Trans. Aerospace and Electronic Sys., vol. 35, no. 3, pp. 892-902, July 1999.

[4] K. M. Kaplan, Q. Le, and P. Molnar, "Maximum likelihood methods for bearings-only target localization," Proc. IEEE ICASSP, vol. 5, pp. 3001$3004,2001$.

[5] X. Sheng and Y. Hu, "Maximum likelihood wireless sensor network source localization using acoustic signal energy measurements," submitted to IEEE Trans. on Sig. Proc., 2003.

[6] D. Li and Y. Hu, "Energy based collaborative source localization using acoustic micro-sensor array," EURASIP Applied Sig. Proc., no. 4, pp. 321-337, 2003

[7] L. Kinsler, A. Frey, A. Coppens, and J. Sanders, Fundamentals of Acoustics, 4th ed. Wiley, 1999

[8] E. Welsh, W. Fish, and P. Frantz, "Gnomes: A testbed for low-power heterogeneous wireless sensor networks," in IEEE Inter. Sym. Circuits and Systems (ISCAS), May 2003.

[9] Connexions: Micro-controller and Embedded Systems Laboratory, http://cnx.rice.edu/content/col10215/latest/. 